Gut, 1960, 1, 183.

\title{
POST-GASTRECTOMY SYNDROMES: A REVIEW
}

\author{
BY \\ C. F. W. ILLINGWORTH \\ From the University of Glasgow and Western Infirmary, Glasgow
}

Arthur Hurst many years ago enunciated the aphorism "gastro-enterostomy, a disease". He might with much greater justice have scoffed at gastrectomy which, though it eliminates the ulcer, too often creates its own peculiar aftermath of afflictions. Hurst did indeed anticipate our present problem, for in 1913, writing on the unfavourable effects of gastroenterostomy, he described one clear case of what we now call the "dumping syndrome", and moreover he recognized it to be the result of too rapid drainage of the stomach.

The term "dumping" seems to have been applied first by Dr. C. H. Mix, a Chicago surgeon, in a paper entitled "Dumping stomach following gastrojejunostomy", which was published in 1922. His case was a woman aged 40 , referred to him by a physician "who was doing the best he could but that was little because her needs were great". Mix carried out an $x$-ray examination and reported that the bismuth slipped out of the stomach almost as fast as it entered. He drew the conclusion that "Nature never intended to have the stomach connected up with the intestine, just any place". Nature has, alas, been thwarted many times since then, and examples of the post-gastrectomy syndromes now bulk large among the return cases of every surgeon engaged in this field.

There is general agreement that all the various disturbances under consideration are due to the precipitate unloading of food from the stomach into the small intestine. However, the disturbances take different forms in different patients and it is convenient to describe four distinct syndromes, though sometimes they occur together in greater or less degree, namely, (1) the early post-prandial (postcibal) syndrome, (2) bilious vomiting, (3) the late post-prandial or hypoglycaemic syndrome, and (4) the malabsorption syndrome.

The term "dumping syndrome" should logically be applied to all these conditions but is usually restricted to the early post-prandial syndrome. Many writers have regarded bilious vomiting as merely one component of the post-prandial syndrome, and indeed they sometimes occur together. However, most patients with post-prandial symptoms do not vomit, and most patients with bilious vomiting have relatively little post-prandial trouble. Moreover, they may arise from different causes and certainly they respond to different types of operative treatment, so they are best discussed separately.

\section{The Early Post-Prandial Syndrome}

This syndrome develops during or shortly after a meal and is characterized by epigastric distension and discomfort with muscular weakness, drowsiness, and faintness. It persists for half an hour or so and then passes off completely until the next meal. In some cases the relief of symptoms is marked by vomiting of a few ounces of pure bile or the passage of a copious soft stool.

The most notable feature is the feeling of extreme weakness. In the mildest cases the patient when walking or standing after a meal feels light headed, almost as though intoxicated, and a little unsteady in his gait. In more severe cases the limbs feel heavy and weak and there is a compelling urge to lie down. There many be transitory fainting or black out. Sometimes there is a sensation of heat throughout the body with flushing or perhaps a cold sweat. During the attack the pulse rate may rise. The blood pressure may rise or fall.

The early post-prandial syndrome generally appears a few weeks after operation, when the patient is becoming more active and taking larger meals. At that phase it probably occurs in greater or less degree in the majority of gastrectomy cases. During succeeding months the symptoms tend to improve but in a hard core of cases they persist in a disabling form. Occasionally post-prandial symptoms arise for the first time several months or even years after the operation.

FreQuenCY AND INCIDENCE.-In my own series, as reported recently by Duthie (1960), post-prandial symptoms of some sort or other occurred at some time during the post-operative course in nearly $80 \%$ of cases. The commonest feature was epigastric fulness, but as a rule it was of slight degree 
and caused little disturbance. On the other hand the vasomotor symptoms, which occurred in $47 \%$ of cases, caused a good deal of disability and in their more severe forms they could be incapacitating for patients engaged in active occupations. Fortunately, in the majority the symptoms were not severe-even at the worst they tended to be regarded as an improvement on the pre-operative ulcer painand they tended to diminish in severity in the course of six months or a year. However, at the end of six years there remained a hard core of $9.4 \%$ patients in whom severe symptoms persisted.

The Polya type of operation (gastrectomy with gastrojejunal anastomosis) and its various modifications are the procedures most likely to cause trouble, but post-prandial symptoms may occur also after the Billroth I operation (gastrectomy with gastroduodenal anastomosis) and even, rarely, after gastrojejunostomy. It is not known whether the additional step of vagotomy has any significant effect. Study of the literature, which has been reviewed recently by Capper and Welbourn (1955), makes it clear that there is marked diversity of opinion as to the frequency with which the syndromes develop in patients of various clinical types, and also a diversity of opinion as to the effect of different types of operative technique.

There is probably no sex difference. Some writers have found women twice as liable as men, but in my own series the reverse was the case. Nor is there any clear evidence as to whether patients treated originally for gastric ulcer differ from those treated for duodenal ulcer. It is said that patients who have had long-standing severe pain before operation are less liable to post-operative trouble but this is probably because they are more willing to tolerate what they regard as a comparatively trifling disability.

As regards the precise technique of operation, most writers are agreed that the risk of dumping is greatest in those cases where a large amount of stomach has been removed and in those where the stoma is of large size, in other words, where rapid unloading is most likely to occur. There are diverse opinions but little tangible evidence as to the relative liability of cases with antecolic or retrocolic anastomosis or between left-to-right and right-toleft arrangement of the jejunal loop.

PatholOGY.-Every surgeon with experience of operating on cases with severe post-prandial symptoms must have been impressed by the paucity of the pathological changes within the abdomen. The stoma itself rarely presents any abnormality of note. Changes have been described affecting the afferent loop-fixation, scarring, partial obstruction, dilatation-but they are probably due to technical defects in the performance of the gastrectomy and are certainly not present in many patients with severe symptoms. In my own experience it is rare to find any recognizable abnormality of the afferent jejunal loop.

Only the efferent loop presents pathological changes and they are constant and characteristic. This part of the jejunum is somewhat enlarged, perhaps to twice its normal diameter, thick-walled, vascular, congested, heavy with blood. There is no obstruction twisting, or kinking. Distally, these changes extend for perhaps 8 or 10 in. and then gradually diminish. It cannot be doubted that they are the result of irritation by food unloaded precipitately from the stomach. They provide an index of the stress to which this part of the small intestine is subjected after gastrectomy and make it easy to understand how motor excitability, stasis of blood, and osmotic fluid shift may occur in this area after a meal.

Methods of Investigation.-One of the difficulties in finding the cause of the post-prandial symptoms arises from the fact that, like most subjective impressions, they are fickle in occurrence and very liable to be modified or obscured by incidental conditions. For example, the symptoms subside quickly when the patient lies down; if he takes his meals in bed the symptoms do not develop. A patient who gives a history of severe symptoms occurring almost without relief since the time of the gastrectomy may not produce a single symptom if he is admitted to hospital for investigation. This fickleness of occurrence has led some observers to suspect a psychosomatic factor, but it is quite possible that the relief on admission to hospital is due to the diminished bowel motility which every patient experiences during his first few days in hospital.

In order to provoke post-prandial symptoms for the purposes of investigation it is customary to give hypotonic glucose solution by mouth, the usual dose being 100 or $150 \mathrm{ml}$. of $50 \%$ glucose in water flavoured with lemon. Such a meal brings on an attack with fair regularity in a patient liable to the spontaneous attacks provided he remains sitting or standing. It must be remembered, however, that attacks may follow a meal containing no glucose, so the biochemical changes following a glucose meal may not necessarily be essential features of the spontaneous syndrome.

Aetiology of the Early Post-prandial SynDROME. - It seems probable that the two components of the early post-prandial syndrome, the epigastric 
distress and the vasomotor features, are brought about in different ways.

The epigastric fulness and discomfort are almost certainly related to motor disturbance in the small intestine. The vasomotor symptoms have been attributed to a number of different causes, including blood sugar changes, sympathetic stimulation, potassium loss, and falling plasma volume.

There is no doubt that all these processes can occur, but their occurrence is not limited to patients with symptoms, and many workers have expressed the view that they occur just as often and in just as marked a form in patients who are symptom free. For this reason their importance as the cause of the syndrome remains in doubt.

Intestinal Motility.-Many radiological studies have shown that after gastrectomy the stomach empties rapidly and transmission of the meal through the small intestine also is usually very rapid. Sometimes also the meal is transmitted rapidly through the colon.

One of the most recent and most complete of such radiological studies is that by Duthie and McKellar (1960). They examined 29 cases after Polya gastrectomy, using a non-flocculating barium sulphate micro-suspension to which was added 75 g. glucose. They confirmed that the stomach remnant nearly always empties very rapidly. In 20 cases it was empty within 10 minutes. Small intestine hypermotility was evident on screening when the bowel was seen writhing and twisting as the opaque meal passed rapidly to the colon. The time taken for the head of the meal to reach the caecum in about half the cases was 20 minutes or less; in one case the caecum was reached in six minutes. Hypermotility of the colon was also seen, though less frequently.

Another feature, described by several previous workers, is a lack of definition of the small bowel. The mucous pattern is blurred and sometimes small fluid levels are seen. This picture is attributed to dilution of the barium suspension by an excess of fluid, doubtless an osmotic shift of water in response to the hypertonic glucose. There is little doubt that this excess of fluid is an important cause of the hypermotility.

The motility pattern of the small intestine has also been examined by the use of inflatable balloons connected with the kymograph. Glazebrook and Welbourn (1952) made observations by this means, the balloon being introduced through the stomach into the efferent loop of jejunum. They confirmed that after gastrectomy the small intestine shows a great deal of motor activity and in particular there are numerous rapid-frequency waves of contraction similar to normal segmentation waves.
There seems good reason to accept the view that these motility changes are responsible, at least in part, for the epigastric fulness and distress which occur soon after a meal. Possibly the aching heaviness described by many patients is due in part also to vascular congestion of the afferent loop of jejunum.

A few workers have suggested that the intestinal hypermotility is also responsible for the vasomotor features of the early post-prandial syndrome, perhaps by initiating a reflex action and liberating a vasoconstrictor substance such as adrenaline. In support of this view Liljedahl, Mattsson, Pernow, and Wallensten (1959), who studied the small intestine by ciné radiography, claim that peristalsis is livelier in patients with vasomotor symptoms than in those who are symptom free. Duthie and McKellar, on the other hand, could find little difference either in the rate of gastric emptying or the time taken for the meal to reach the caecum, though they noted that hypermotility of the colon was more marked in the group with symptoms. Incidentally, Duthie and McKellar noted that the hypermotility persisted when the patient was put in a supine position, although vasomotor symptoms did not then develop.

Blood Sugar Changes.-It has been known for many years that after gastrectomy, both in patients with symptoms and in those without symptoms, the blood sugar level is subject to wide fluctuations.

Lapp and Dibold in 1933 found that in patients after gastric operations when glucose was given by mouth there was an abrupt rise in blood sugar to an abnormally high level, followed by a sharp fall to within the hypoglycaemic range. They noted also that comparable changes could be produced in normal subjects giving the glucose through a tube directly into the jejunum. Similar findings have been reported by many writers since that time.

There is, of course, a good deal of individual variation, and in some gastrectomy cases the results of the glucose tolerance test lie within the normal range. More commonly, however, the blood sugar at $30 \mathrm{~min}$. or $45 \mathrm{~min}$. will rise to above $150 \mathrm{mg}$. per $100 \mathrm{ml}$. and it may rise to 250 or even to over $300 \mathrm{mg}$. It tends to maintain this high level for an hour or more and then falls abruptly to $50 \mathrm{mg}$. or lower.

The customary explanation of the early rapid rise of blood sugar is that it is due to unduly rapid absorption from the jejunum consequent on the precipitate gastric emptying. Indeed, Smith and his colleagues have used the rate of sugar elevation as an index of the rate of gastric emptying. It is possible, however, that some part of the rise in blood sugar may be due to the action of adrenaline, or 
perhaps due to faulty utilization of the circulating sugar.

The conditions governing the late hypoglycaemic phase will be considered later, in connexion with the syndrome which occurs at that time.

It has long been known that the early postprandial syndrome is not related to hypoglycaemia, which occurs much later. The early syndrome develops precisely at the time when the early rise in the level of the blood sugar is beginning to make its appearance. Glaessner (1940), who was the first to draw attention to this coincidence, attributed the symptoms to "hyperglycaemic shock", and other writers since that time have attempted to relate the two phenomena, either directly or indirectly. There is, however, a good deal of evidence against this view.

The blood sugar changes occur in gastrectomy patients who are without symptoms as well as in those with post-prandial symptoms, and there is diverse evidence as to whether the sugar changes are greater in the one group than the other.

Smith, Fraser, Staynes, and Willcox (1953), in an investigation of 26 patients subjected to gastrectomy after various types of meal, found a definite correlation between the steepness and extent of the rise in blood sugar and the severity of the symptoms; on the other hand, most other workers have been unable to confirm this finding. In my own unit, Duthie (1959), who made a study of 40 cases after Polya gastrectomy, found that the mean blood sugar levels in 20 patients with vasomotor symptoms were no different from those in the 20 who were symptom free. Nor was there any difference in the rate of rise of the blood sugar. In his series also there was no correlation between the blood sugar rise and either the fall of plasma volume or the electrocardiographic changes.

It is perhaps even more informative to study in detail individual records of blood sugar levels and to compare them with the severity of the symptoms with which they were associated. The paper by Schechter and Necheles (1949) is convincing in this respect. These workers studied 11 cases after administering $100 \mathrm{~g}$. glucose in $3 \%$ solution by the mouth. Four of these patients showed no undue degree of elevation of the blood sugar yet they developed vasomotor symptoms, of moderate severity in three, severe in the remaining one. Two of the patients showed marked hyperglycaemia yet remained symptom free.

Perhaps the most emphatic evidence against hyperglycaemia as the cause of the early post-prandial symptoms is provided by two complementary types of observation. It has been shown (Schechter and Necheles, 1949; Duthie, 1959) that hyperglycaemia of comparable degree produced by infusing glucose intravenously causes no symptoms. And it has also been shown that typical early post-prandial symptoms can be produced by manoeuvres which cause no hyperglycaemia, for example, by giving laevulose (Schechter and Necheles) or mannitol (Duthie, 1960) which are absorbed and metabolized too slowly to affect the blood glucose level, or by giving a protein meal (Borgström, 1957), and even by distending the jejunum with a balloon (Machella, 1950; Glazebrook and Welbourn, 1952).

Sympathetic Stimulation.-Irvine in 1948 in a study of 24 cases noted that the early post-prandial symptoms are related to the bulk of a meal rather than to its content of glucose and supported the view that distension of the gut might be responsible by initiating a nervous reflex. Unlike previous workers, who had blamed vagus stimulation, he suggested that stimulation mediated by sympathetic nerves might be responsible. The weakness, giddiness, perspiration, and faintness are not unlike the effects produced by adrenaline and the rise in pulse rate and blood pressure which are observed in some cases might be produced in the same way. Indeed part of the rise in blood sugar level also could be attributed to release of adrenaline. Other workers have supported this view; for example, Smith and his colleagues (1953) who noted that the early postprandial symptoms could be reproduced by injecting adrenaline, while Pulvertaft (1954) noted an increase in blood catechol levels, though they did not correlate well with the onset of symptoms.

Electrocardiograph studies give some limited support to this view. Many workers (Smith, 1951), Roberts, Randall, Farr, Kidwell, McNeer, and Pack (1954), Pulvertaft (1954), Peddie, Jordan, and De Bakey (1957), and Duthie (19,59) have carried out such studies. They show that changes in the electrocardiograph tracing begin five to 15 minutes after a meal and attain a maximum at 20 to 30 minutes. There is an increase in heart rate, varying from a few beats to 50 a minute. There is lowering or flattening of the $T$ wave and a $U$ wave may make its appearance or become more pronounced if already present. The S-T segment may be elevated or depressed and there may be some peaking of the $P$ wave.

Although these or similar features have been reported by all those who have studied the matter there is no agreement as to their significance. They have been attributed to potassium deficiency (Smith, 1951) and to coronary arterial insufficiency (Roberts et al., 1954). Pulvertaft (1954) noted that they were related to the rate of absorption of sugar from the intestine. Duthie (1959), who has made a special study of the problem, has been unable to find a 
correlation between these changes and the rise in blood pressure or the fall in plasma volume or the severity of symptoms.

Thus, there is little disposition to regard a sympathetic stimulation as the major cause of the post-prandial symptoms though it may well be a contributory factor. It is possible that the falling blood volume, acting through aortic pressoreceptors, stimulates the outpouring of adrenaline and this may perhaps be responsible for the tachycardia and the maintenance of blood pressure as well as some of the symptoms.

Potassium Loss. - The idea that the muscle weakness and drowsiness which occur as part of the early post-prandial syndrome might be due to a temporary deficiency of potassium was first suggested by Smith (1951). He studied six patients after standard meals and high-protein meals and also after they had been given hypertonic glucose solution. He noted that whereas in normal control patients the potassium level remained unaltered, in patients liable to post-prandial symptoms it regularly fell to the lower limit of normal. When potassium was given intravenously, although the other components of the syndrome were unaffected, the weakness and fatigue were controlled. He suggested that the fall in potassium might be due to the release of an adrenaline-like substance or to unduly rapid absorption of carbohydrate, leading to rapid deposition of glycogen with binding of potassium inside the glycogen-storing cells.

Certainly the symptoms are quite like those of potassium deficiency and moreover the electrocardiographic changes, as have already been discussed, might well be due to the same cause. The idea has been criticised for the reason that the fall in serum potassium is not pronounced and such fall as there is tends to become evident rather late after the meal, when the symptoms are beginning to abate. However, it must be acknowledged that the serum potassium is not a true index of the level of potassium within the cells.

Fall of Plasma Volume.-One of the most consistent and notable changes to be observed in the early post-prandial phase is a reduction in the volume of blood in circulation.

Roberts and his colleagues (1954) seem to have been the first to draw attention to this matter. They examined 10 cases after total gastrectomy and four after subtotal gastrectomy, using the Evans blue method, and noted a marked fall in plasma volume which developed within 10 minutes after a meal of $50 \%$ glucose and reached a maximum between 30 and 40 minutes. Similar changes have been observed by several subsequent workers (Amdrup and Jørgensen, 1957; Hinshaw, Joergenson, Davis, and
Stafford, 1957; Peddie et al., 1957; Everson and Abrams, 1958; Weidner, Scott, Bond, and Shull, 1959).

The fall in plasma volume, as estimated by this method, averages about 5 or $6 \%$ but in severe cases it may exceed $10 \%$ or even $15 \%$. A similar fall has been observed after a meal of fructose or mannitol or a meal rich in proteins.

There is general agreement that the fall in plasma volume is due to osmotic withdrawal of fluid from the plasma into the jejunal lumen as a result of the hypertonic meal. This view receives confirmation from the observation by Amdrup and Jørgensen that the fall of volume is related to the amount of fluid seen in the lumen.

Like all the other post-gastrectomy developments we have reviewed the fall of plasma volume is observed in patients without symptoms as well as in those with symptoms. Some observers have found a correlation between the extent of plasma volume change and the symptoms. Thus Amdrup and Jørgensen found the fall almost directly related to the intensity of symptoms, and Le Quesne and his colleagues came to a similar conclusion. Other workers (Peddie et al., 1957; Everson and Abrams, 1958) were unable to confirm this result.

Duthie (1960) has studied the question in my department, using radio-iodinated human serum albumin as the marker substance. He investigated 20 gastrectomy patients with post-prandial symptoms and 20 who were symptom free. The maximum fall in plasma volume averaged $8.3 \%$ in the former group and 7.0 in the latter; the difference was not statistically significant. Moreover, he found that severe degrees of fall in plasma volume (more than $10 \%$ ) were but little more frequent in patients with symptoms than in the symptom free.

Additional Features.-The observations reported above make it clear that many gastrectomy patients present a complex vasomotor post-prandial disturbance of which the central feature is the fall of plasma volume due to osmotic fluid shift into the intestine. However, all the changes which have been observed occur not only in patients with postprandial symptoms but also in those who are symptom free, and in the opinion of most observers they are just as marked in the latter group as in the former.

It is clear therefore that there must be some factor of individual susceptibility or some other unidentified factor which determines which patients will develop symptoms.

Le Quesne, Hobsley, and Hand (1960) have demonstrated the existence of an individual susceptibility to falling plasma volume by applying 
pneumatic tourniquets to the thigh. When inflated to obstruct the venous return, they abstract $400-700 \mathrm{ml}$. of blood from the circulation in the course of 15 to 20 minutes, which is closely comparable to the plasma volume change after a meal. In patients liable to post-prandial symptoms this procedure led to faintness and drowsiness whereas in other patients it was tolerated without noticeable effect.

It is indeed true that the plasma volume loss is hardly sufficient to account for symptoms in robust subjects (the loss is no more than every blood donor tolerates willingly) and the possibility of some additional factor must be considered.

Hinshaw and his colleagues believe that the factor concerned is a failure of the homoeostatic response to diminished plasma volume. The normal response should be a peripheral vasoconstriction, and this, they find, occurs in gastrectomy patients who are without symptoms. In those liable to post-prandial symptoms, on the other hand, digital plethysmography shows that instead of vasoconstriction there is an increase in the peripheral blood flow, sometimes up to 12 times the normal.

Another possible factor is pooling of blood in stagnant backwaters of the circulation, most probably in the vascular jejunum and its mesentery and the portal area as a whole. The usual methods of estimating plasma volume will not show such stagnation, for they measure the concentration of dye (or radioactive iodine) bound to the albumin and, apart from the normal disappearance rate, this concentration is altered only by escape of the nonalbuminous fluid from the circulation.

Duthie (1959) has sought to demonstrate blood stasis by a modification of the plasma volume estimation, in which Evans blue is given in intermittent doses, on the basis that later doses might not mix with blood in stagnant areas, and in two patients with severe dumping he produced some evidence of blood stasis by this means. A more refined technique is to estimate the plasma loss in the usual way and also the packed cell volume (P.C.V.) at intervals after a meal. The recorded P.C.V. is then compared with the figure calculated from the initial P.C.V. as it would be modified by the plasma loss. In such observations on 35 cases Duthie found a striking correlation between blood stasis and symptoms.

Medical Care of Patients with Post-prandial SYNDROME.-In view of what is known about the early post-prandial syndrome, rational treatment should be directed to correcting the precipitate emptying of the stomach and minimizing the effects of the fall in plasma volume. The treatment em- braces advice on diet and medication, attention to the general health, and adjustment of the way of life. Spontaneous improvement takes place in most cases during the first year or so, and it is reasonable to suppose that medical care will assist this process.

Every gastrectomy patient should be given proper advice about his diet, for the conditions under which digestion proceeds are entirely different and a diet designed to mitigate the pain of ulcer may be entirely unsuited to the post-operative state. Sometimes indeed milk and milk products seem to precipitate the post-prandial symptoms.

After gastrectomy it is essential to reduce the bulk of the meal. Different patients vary, but each should find his own capacity and not exceed it. To do so it is often a good plan to eschew bulky soups and sweets, and be content with a single course. Bulky carbohydrates, such as potatoes, should be limited and cooked fats should be reduced, though butter may be well tolerated. Though small in bulk the choice of food can be fairly wide and the meals can be made very appetizing to patients inured to long years of an ulcer diet. Meat is usually well tolerated, all kinds of vegetables, salads, and fruits, and even pickles, sauces, and condiments.

Medication should be reduced to the minimum after gastrectomy. There is no need for alkalies, antacids, and adsorbents and they may be positively harmful.

In addition to this kind of regimen, attention must be paid to the general health. Patients with postprandial symptoms are often anaemic and under weight, and while these are usually the results of the syndrome, if they can be corrected the symptoms may be improved. Anaemia may respond to iron but if it is severe a transfusion of blood is desirable. For weight loss an anabolic steroid preparation may be tried. It has been claimed that good results may sometimes be obtained from insulin in small doses, administered just before the main meal.

It would seem rational to slow down the bowel motility by the use of ganglion blocking agents, but such treatment has proved disappointing. Finally, as an auxiliary to medical treatment, patients who can do so should be advised to lie down for half an hour after meals. This may prevent the attacks and will certainly diminish their severity.

Surgical Treatment.-Many different operations have been advocated in the past, according to differing views on the aetiology of the syndrome. On the theory that an obstruction of the afferent loop was responsible it seemed rational to relieve this obstruction by making an anastomosis between the afferent and efferent loops. As has been the fate of so many suggested treatments for this obscure 
disease, the early results seemed promising but continued experience has not substantiated them. In the same way the Roux-Y operation, while believed to be effective for bilious vomiting, gives no relief to the vasomotor features of the post-prandial syndrome. On the basis of present-day views on the aetiology the rational treatment would be to diminish precipitate emptying either by enlarging the capacity of the stomach or by reducing the size of the outlet.

Attempts have been made to enlarge the capacity by transplanting an isolated segment of colon so that it lies between the gastric remnant and the jejunum. Few surgeons have published their exexperience of this operation so that there is little reliable evidence about it. Probably it can be inferred that few surgeons have been sufficiently impressed by their experience to be encouraged to repeat it.

On the whole, better results seem to have been obtained from methods designed to diminish the rate of outflow tract from the stomach, and at the present time this type of operation is the procedure of choice for severe cases of the early post-prandial syndrome.

The most satisfactory method is to convert to the Billroth I gastroduodenal anastomosis, which not only reduces the size of the stoma and introduces the resistance of the relatively narrow duodenum, but also gives benefits related to the absorption of fat (see below). If conversion to Billroth I is technically too difficult (in my experience this is rare) the alternative is to dismantle the Polya stoma, and make a new anastomosis of smaller size.

The steps of the conversion to Billroth $I$ are as follows:-

After full exploration to exclude other lesions, the first step is to free the region of the gastrojejunal anastomosis from all adhesions and, in the case of a retrocolic anastomosis, to free it from the mesocolon. Next, the stomach remnant must be mobilized freely to enable it to be brought across to the duodenum without tension. On the lesser curve, the stomach should be mobilized completely up to the oesophageal orifice. The greater curve should also be freed almost to the fundus, but at least one of the short gastric vessels must be retained to maintain the circulation. Great care should be taken to avoid damage to the spleen or its pedicle, which might prejudice this blood supply.

When this has been done, attention is directed to the duodenal stump. It is exposed by dividing the peritoneal adhesions over it and dissected free. The second and third parts of the duodenum, along with the pancreas, are also sponged forwards to ensure greater mobility. The duodenum is now opened either at its blind end or by a longitudinal incision extending to the second part.

At this stage attention is turned again to the gastrojejunal anastomosis. Sometimes it is satisfactory to undo the anastomosis and repair the defect in the jejunum with an infolding stitch. More often this would leave an irregular, dog-eared and narrowed jejunum and it is better to resect a few inches cleanly and restore continuity by end-to-end anastomosis.

The open end of the gastric remnant must then be reduced in size by a continuous suture starting from the lesser curvature, the aim being to make the opening in the stomach roughly commensurate with that previously made in the duodenal stump. Generally about $3 \mathrm{~cm}$. suffices. Finally, this opening is united to the duodenum. All the suturing must of course be done with great care, and particularly so for the final stage, the gastro-duodenal anastomosis. The aim is to obtain exact apposition with neat stitching, to minimize the risk of oedema and obstruction of the stoma during the ensuing few days. If this is done, the post-operative course should be unremarkable.

\section{BiLIous Vomiting}

As has already been said, bilious vomiting may occur as part of the early post-prandial disturbance or as an isolated phenomenon. It varies also in severity. One patient may complain of little except that from time to time he feels a little nauseated, vomits a few mouthfuls of pure bile, and is then once more all right. Another patient has attacks of epigastric discomfort, building up for a few hours and culminating in the regurgitation of a large quantity of bilious fluid. In such cases there may be other post-prandial symptoms, much weight loss, and severe disability.

The cause of the bile vomiting is not fully understood. There is, of course, no difficulty in explaining why the vomit contains bile and not food, for the vomiting usually occurs an hour or so after a meal and by that time all food has left the stomach. Nor is it difficult to explain why so much bile, in such a pure state, should appear so long after a meal; presumably the cholecystokinin mechanism for emptying the gall bladder acts more slowly since food does not enter the duodenum. The real difficulty is to explain why, since the meal has passed normally towards the lower reaches of the bowel, there should be some impediment to the transmission of bile in the same direction.

The explanation generally favoured attributes this symptom to transient obstruction of the afferent loop, which gradually becomes distended with bile 
and then suddenly releases it into the stomach. In some cases the cause of obstruction can be demonstrated at operation. This is particularly so where the gastrectomy has been of antecolic type with a large dependent afferent loop. In other cases no organic obstruction can be demonstrated at operation and it must be supposed that the obstruction is caused by kinking or rotation of the afferent loop when the stomach is distended with food. Various methods of operation have been devised for the treatment of bilious vomiting. They have been reviewed by Capper and Welbourn (1955). Where the vomiting is a relatively insignificant feature of a post-prandial syndrome in which the vasomotor symptoms predominate, it seems probable that the most satisfactory treatment, as already discussed, is to convert the anastomosis to one of Billroth I type. On the other hand, where the bilious vomiting is the principal cause of disablement, a more satisfactory method is to create a Roux-Y-shunt. The afferent loop is divided just short of the anastomosis. The end abutting on the anastomosis is closed. The proximal end is then inserted, end-to-side, into the efferent loop some 6 or 8 in. further distally. Conyers, Hall, Laing, Pulvertaft, and WillsonPepper (1960) have recently reported their experience of 54 cases treated in this way, with 19 excellent results and only two which could be classified as unsatisfactory.

\section{The Late Hypoglycaemic Syndrome}

As its name indicates, this syndrome occurs late after a meal (two to three hours) and coincides with the depression of the blood sugar level which occurs in some gastrectomy patients at that stage. Although hypoglycaemia is fairly common after gastrectomy the hypoglycaemic syndrome is generally regarded as rare. Perhaps this is because many patients learn to recognize the premonitory symptoms and prevent the attacks by taking food.

The attacks are similar to those of hypoglycaemia due to other causes and are characterized by tremor, giddiness and excitement, sweating and muscular weakness. In severe cases they culminate in unconsciousness.

Although the relation to hypoglycaemia seems to be established there are some puzzling features. It is not yet really certain why the hypoglycaemia occurs. The usual explanation, that it is due to a delayed and excessive output of insulin in response to the initial hyperglycaemia, does not accord with the observation that no similar output follows hyperglycaemia induced by intravenous glucose. According to Smith and his colleagues (1953) the essential abnormality lies in oversensitivity to insulin, which aggravates the hypoglycaemia and makes it unduly prolonged.

Patients who suffer from the hypoglycaemic syndrome do not necessarily give an abnormal response to a glucose tolerance test carried out during an interval between attacks. It looks as though some additional factor is necessary to provoke an attack. The essential diagnostic step is to examine the blood sugar during an attack, when it will certainly be below $50 \mathrm{mg} . \%$ and may be as low as $20 \mathrm{mg} . \%$.

Dietetic treatment usually suffices to prevent attacks. The patient must be advised to avoid rapidly assimilable carbohydrates in order to prevent the extremes of blood sugar fluctuation caused by rapid absorption. However, despite this general recommendation the patient may carry sugar to use in emergencies when an attack seems imminent. In severe cases it may be necessary to advise conversion to a Billroth I anastomosis.

\section{The Malabsorption Syndrome}

Under this generic title we include various disturbances after gastrectomy due to impaired absorption of foodstuffs, iron, and vitamins. They may occur as isolated phenomena but usually, especially when severe, they occur as concomitants of severe post-prandial syndromes.

Loss OF WEIGHT.-It is a common experience that the majority of patients after gastrectomy fail to maintain their "tabular" weight (the weight appropriate, according to accepted tables, for their height and age), and many fall far below that level. Anderson, Gunn, and Watt (1955), in a review of nearly 500 cases from my unit, found that $55 \%$ of the men and $65 \%$ of the women were more than $5 \%$ underweight. More recently Duthie (1960) in a smaller series of 89 cases found that an even higher proportion $(67 \%)$ had lost some weight. He confirmed the general view that the weight loss tends to be greatest in patients with severe post-prandial symptoms.

Apart from extraneous causes, such as tuberculosis, the weight loss may be due to three causes, reduced intake, vomiting, and impaired absorption. Reduced intake is of especial importance in those patients who find they have to limit the bulk of their meals in order to prevent post-prandial disturbances. Sometimes also such patients are nauseated, and their appetite is affected in consequence. A particularly difficult problem is seen occasionally in elderly patients with persistent impairment of general health, who become depressed and apathetic and anorexic, and go downhill from simple starvation. 
However, the great majority of gastrectomy patients have good appetites and for them the cause of the weight loss is to be found in some defect of absorption, particularly of fats. This is indeed obvious from the naked eye character of the stool, which may be bulky, pale, and greasy. It is readily confirmed by estimation of the total fat content.

There are several possible causes of the malabsorption.

(1) Mistiming of the bile and pancreatic secretion may be partly responsible, for normally bile and pancreatic juice are secreted in response to the presence of food in the duodenum and their secretion is slow to match the normal emptying of the stomach. After gastrectomy the secretory response is poor and sluggish, so emulsification and digestion are impaired.

(2) It has been suggested that the diminished gastric acidity after gastrectomy may lead to impaired pancreatic secretion and vagotomy also has been claimed to have a similar effect (Butler, 1960).

(3) The attention focused in recent years on idiopathic steatorrhoea has led to the suggestion that the steatorrhoea after gastrectomy might have a similar cause. Shiner and Doniach (1958) have examined portions of jejunal mucosa removed at biopsy from 11 gastrectomy cases. The appearance was entirely normal in nine cases, but in the remaining one there was marked atrophy of the villi with an increase in total thickness of the mucosa. Joske and Blackwell (1959) made similar findings in four patients who suffered from malabsorption after gastrectomy.

It seems doubtful, however, if jejunal biopsy material can give valid information in these cases, for we know from simple examination at operation that the efferent loop of jejunum, from which the biopsy tissue is removed, is far from representative of the small intestine as a whole.

(4) It seems probable that the main cause of the weight loss is the obvious one, namely that absorption of food products from the small intestine is impaired as a result of intestinal hurry. Radiological studies show that the barium commonly reaches the caecum within 15 minutes, and while the barium meal is doubtless a fallacious index of intestinal movement under normal conditions there can be no doubt, from a mass of clinical evidence, that the passage of ordinary food through the intestine may be very rapid.

Support for this view is given by observations on the absorption of radioactive fat. When fat tagged with radioactive iodine is given by mouth to patients after gastrectomy, the blood level during the first hour rises at the normal rate, but the total absorption, as measured from the amount of radio2 active fat excreted in the stools, may be far below the normal.

The treatment should depend upon the severity of the weight loss. In minor degrees, that is to say less than $10 \mathrm{lb}$. below tabular weight, no treatment is necessary. Indeed the patient should be reassured and congratulated, for it makes for general fitness and also, as Walker, Watson, and Watt (1958) have shown, it may reduce the liability to coronary disease. In more severe degrees of weight loss, the first indication is to exclude extraneous causes, such as tuberculosis, malignant disease, intestinal fistula. A check should be made of the food intake. Postprandial disturbances causing anorexia or vomiting may require treatment. Insulin has been advised to improve the appetite and ensure utilization of the sugar spilled over during the hyperglycaemic phase. Anabolic steroids may be helpful in severe anorexia. Rarely a gluten sensitivity may be found responsible.

However, in most cases of severe weight loss if ordinary dietectic remedies are unavailing and if all other causes have been excluded the question of further operation should be considered. On the basis that intestinal hurry is the factor responsible the rational treatment is to convert to the Billroth I anastomosis, and this is in fact an effective method of relieving the weight loss.

ANAEMIA.-Hypochromic anaemia is common after gastrectomy. Anderson et al. (1955), using the Sahli method, found that over $40 \%$ of the women patients had haemoglobin levels below 20 , while about $20 \%$ had a level below 70 . The tendency to anaemia is much less in men.

In some such cases, continued blood loss is one of the factors involved. Most of Duthie's male patients with anaemia gave a history of bleeding haemorrhoids, and probably the special liability of women to anaemia is due in part to the menstrual loss. The possibility of persistent occult bleeding from erosions in the vicinity of the anastomosis must be considered (Witts, 1956). However, this is clearly not the only factor, and in the majority of cases there is also some defect in the intake of iron due, for example, to anorexia, unsuitable diet, vomiting, or in its absorption Smith (1956).

As in so many other aspects of the post-gastrectomy syndromes, the question at issue raises problems concerned with physiology and once again it focuses attention on the gaps in our knowledge of the normal process. It is generally agreed, based on animal experimentation, that the main site for absorption of iron is in the duodenum and upper jejunum, so it might be supposed that the anaemia after gastrectomy might be due to the short circuiting of this segment by the gastrojejunal anastomosis. 
However, Duthie (1959) has shown, by studies on two patients converted from the Polya to the Billroth, and two patients converted from Billroth to Polya, that the absorption of iron is in no way different in the two types of anastomosis.

The reduced gastric acidity after either form of gastrectomy may well be a factor leading to impaired iron absorption, but it seems likely that the main factor is the same as is responsible for the weight loss, namely, intestinal hurry. This is confirmed by the common finding that the absorption of inorganic iron is not impaired and may even be enhanced, provided it is given in small doses at frequent intervals. Further confirmation is given by the observation of Baird, Blackburn, and Wilson (1959) who showed that the absorption is reduced if the iron is given with a full meal, when intestinal hurry may be presumed to be the factor responsible. In the treatment it is necessary to remember that anaemia is easier to prevent than cure. It is important to give the patient a good start, so the haemoglobin level should be checked before he leaves hospital and if there is a serious deficiency blood transfusion should be performed.

Iron should be given with caution, for excessive doses readily cause diarrhoea. The best method, therefore, is to give small doses over long periods. On his return home after gastrectomy the patient should take iron continuously for a few months. Thereafter the haemoglobin level should be checked from time to time. Since many patients without other symptoms are apt to disregard the minor effects of anaemia it is a good plan to recommend that in any case at each anniversary of the operation iron medication should be given for a month or so. Where anaemia develops despite these precautions a search should be made for sources of hidden blood loss, and the appropriate treatment carried out.

Macrocytic anaemia is well recognized as a late sequel to total gastrectomy and in recent years a few cases have been reported after partial gastrectomy (Blake and Rechnitzer, 1953; Girdwood, 1956). The anaemia is due to faulty absorption of vitamin $B_{12}$ and makes its appearance only after an interval of several years, owing to the presence in the liver of large stores of the vitamin. Girdwood (1960) has shown that the absorption of folic acid is normal. In the treatment, administration of vitamin $B_{12}$ is quickly effective.

Malabsorption of Vitamins.-Apart from $B_{12}$, occasional cases have been reported of late symptoms due to failure in the absorption of other members of the vitamin B complex. They are so rare that the possibility of additional causes must be suspected, for example, a dietetic deficiency or a gastro-colic fistula or an intestinal cul-de-sac. Where there is no such cause, the symptoms yield rapidly to administration of the vitamin complex.

\section{REFERENCES}

Amdrup, E., and Jørgensen, J. B. (1957). Acta chir. scand., 112, $307,313$.

Anderson, C. D., Gunn, R. T. S., and Watt, J. K. (1955). Brit. med.

Baird, I., M., Blackburn, E. K., and Wilson, G. M. (1959). Quart. J. Med., 28, 21.

Blake, J., and Rechnitzer, P. A. (1953). Ibid., 22, 419.

Borgström, S. (1957). Acta chir. scand., 113, 426.

Butler, T. J. (1960). Gut, 1, 55.

Capper, W. M., and Welbourn, R. B. (1955). Brit. J. Surg., 43, 24.

Conyers, J. H., Hall, R. A., Laing, D., Pulvertaft, C. N., and WillsonPepper, J. K. (1960). Ibid.. 47, 533 .

Duthie, H. L. (1959). Ch. M. Thesis, University of Glasgow.

- (1960). Scot. med. J., 5, 127.

, and McKellar, N. J.'(1960). Brit. J. Radiol., 33, 171

Everson, T. C., and Abrams, B. (1958). Ann. Surg., 148, 94.

Girdwood, R. H. (1956). Quart. J. Med., 25, 87.

(1960). Scot. med. J., 5, i0.

Glaessner, C. L. (1940). Rev. Gastroenterol., 7, 528.

Glazebrook, A. J., and Welbourn, R. B. (1952). Brit. J. Surg.,

Hinshaw, D. B., Joergenson, E. J., Davis, H. A., and Stafford, C. E. (1957). A.M.A. Arch. Surg., 74, 686.

Hurst (A. F. Hertz) (1913). Ann. Surg., 58, 466.

Irvine, W. T. (1948). Brit. med. J., 2, 514.

Joske, R. A., and Blackwell, J. B. (1959). Lancet, 2, 379.

Lapp, F. W., and Dibold, H. (1933). Klin. Wschr., 12, 547.

Le Quesne, L. P., Hobsley, M., and Hand, B. H. (1960). Brit. med.J., 1,141 .

Liljedahi, S.-O., Mattsson, O., Pernow, B., and Wallensten, S. (1959). Acta chir. scand., 117, 206.

Machella, T. E. (1950). Gastroenterology, 14, 237.

Mix, C. L. (1922). Surg. Clin. N. Amer., 2, 617.

Peddie, G. H., Jordan, G. L., and De Bakey, M. E. (1957). Ann. Surg., 146, 892.

Pulvertaft. C. N. (1954). Lancet, 1, 325.

Roberts, K. E., Randall, H. T., Farr, H. W., Kidwell, A. P., McNeer, G. P., and Pack, G. T. (1954). Ann. Surg., 140, 631.

Schechter, S. E., and Necheles, H. (1949). Gastroenterology, 12, 258.

Shiner, M., and Doniach, I. (1958). In World Congress of Gastroenterology, Washington (U.S.A.), p. 586.

Smith, M. D. (1956). Proc. roy. Soc. Med., 49, 868.

Smith, W. H. (1951). Lancet, $2,745$. Med., 22,381.

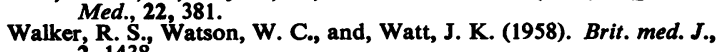
2, 1438 .

Weidner, M. G., Scott, H. M., Bond, A. G., and Shull, H. J. (1959). Gastroenterology, 37, 188.

Witts, L. J. (1956). Anaemia and the Alimentary Tract. Royal College of Physicians, Edinburgh. Publ. No. 7. 